\title{
Vie brève des antibulles
}

\author{
Stéphane Dorbolo ${ }^{1(*)}$, Étienne Reyssat ${ }^{2}$, Nicolas Vandewalle ${ }^{1}$, David Quéré ${ }^{2}$ \\ 1. GRASP, Université de Liège, Belgique \\ 2. Laboratoire des fluides organisés, UMR 7125 du CNRS, Paris
}

\begin{abstract}
Une antibulle est une goutte liquide couverte d'une mince pellicule d'air (ou d'huile) et plongée dans un bain du même liquide. Cet objet est potentiellement intéressant pour l'encapsulage et le transport d'espèces liquides. Dans cet article, on présente et discute les mécanismes de vieillissement et de mort des antibulles, qui se révèlent instables. Elles diffèrent en ceci des bulles de savon (métastables), par suite de l'absence de forces électrostatiques dans le film d'air.
\end{abstract}

Une bulle est à l'origine (latine) une bulla, c'est-à-dire une boule; boule de métal d'abord, attachée à un sceau, et par extension ce sceau lui-même. Les bulles du pape sont (au prix d'une nouvelle extension) les décrets pontificaux marqués d'un tel sceau - et quand elles sont comminatoires, on dit du pape qu'il a fulminé des bulles. Rien d'aussi foudroyant dans les autres acceptions du mot : une bulle est à la fois une petite cavité gazeuse dans un liquide (du gaz carbonique dans une eau pétillante, par exemple), et une mince pellicule savonneuse dans l'air - bulle de savon soufflée par les enfants, ou motif d'admirables peintures de Chardin et de Manet. Nous discutons ici du négatif de cet objet : l'antibulle sera, hors du contexte pontifical, où ce terme désignait la bulle d'un antipape (du temps où ils existaient) [1], une mince pellicule d'air englobant une goutte d'eau, le tout dans l'eau [2]. Nous nous demandons d'abord comment engendrer de tels objets ; puis nous discutons leur vie et leur mort, en les comparant à celles des bulles de savon.

\section{Générateurs d'antibulles}

La méthode la plus simple pour obtenir une antibulle consiste à verser une eau savonneuse dans un bain de même nature (figure 1). On remplit au préalable un récipient à ras bord, assez délicatement pour obtenir une surface nette, sans bulles. Puis on dépose à la surface une goutte de la même eau : à cause de la présence de tensioactifs, elle flotte un moment avant de fusionner [3]. On peut d'ailleurs pérenniser cette situation en faisant vibrer le bain, comme l'ont montré récemment Couder et al. [4]. Mais même sans cela, et en ajustant patiemment le débit d'alimentation, on parvient à produire une goutte flottante d'un centimètre de diamètre. En propulsant ce globule sous l'eau avec un jet, on crée une antibulle. Un moyen plus efficace encore consiste à envoyer le jet directement dans le bain, avec assez de vitesse pour qu'il entre gainé d'air dans l'eau ; la déstabilisation de ce cylindre par l'instabilité de Rayleigh-Plateau (celle-là même qui transforme le jet d'un robinet en gouttes) engendre alors toute une flottille d'antibulles (figure 1).

À cause de sa coquille d'air, une antibulle remonte dans le bain, poussée par Archimède. De la vitesse de remontée, on déduit d'ailleurs très simplement l'épaisseur de la coquille [5] : typique-

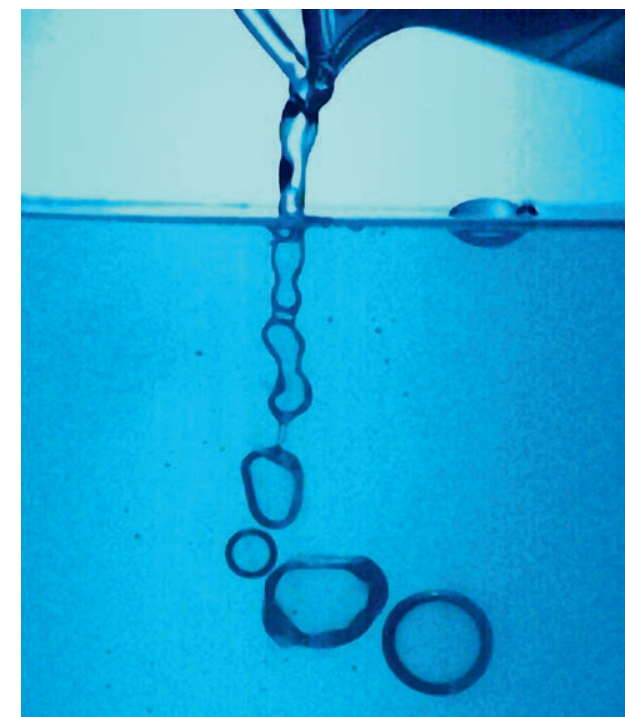

Fig. 1. Photographie sous-marine montrant la génération d'antibulles centimétriques par un jet (on distingue au-dessus du niveau d'eau le bec verseur du bécher). La frontière foncée, autour de chaque antibulle, traduit la présence d'un film d'air.

ment 5 microns, valeur tout à fait comparable à l'épaisseur du film d'eau dans une bulle classique. Pour éviter cette remontée (fatale à l'antibulle, quand elle arrive à la surface), on sale la solution incidente et on place au fond du récipient du glycérol, plus dense et soluble dans l'eau : I'antibulle salée commence par couler, puis s'arrête à l'endroit de ce milieu stratifié qui a sa densité. On peut alors l'observer en toute quiétude.

Une antibulle ainsi fabriquée sera centimétrique, et son aspect brillant provient d'un effet de réflexion totale de la lumière sur le film d'air (figure 2a). La forme est globalement celle d'une sphère, dont la surface est très supérieure à celle d'une cavité d'air de même volume que le film : comme une bulle de savon, notre objet ne sera donc (dans le meilleur des cas) que métastable. On observe aussi un léger renflement au pôle Nord, à cause de la tendance de l'air à remonter, et à s'accumuler à cet endroit. Des franges d'interférence traduisent enfin des variations d'épaisseur du film. Ces différentes observations indiquent un amincissement du film d'air au bas du globule, ce que confirme le mouvement des franges d'interférences et le fait que l'éclatement du film d'air s'amorce en général au pôle Sud. 


\section{Drainage}

La durée de vie d'une antibulle est en général de plusieurs minutes [5]. Certes, elle ne subit pas la cause principale de vieillissement d'une bulle banale (l'évaporation), mais elle est comme elle soumise au drainage : l'air remonte le long de la coquille (ou dit autrement, le globule intérieur s'enfonce lentement dans le film d'air qui le supporte). La cinétique du drainage devrait être dictée par un équilibre entre friction visqueuse (dans le film) et poussée d'Archimède. En notant $\rho$ la masse volumique de l'eau, $g$ l'accélération de la gravité, $\eta$ la viscosité de l'air et $\varepsilon$ l'épaisseur du film, cet équilibre s'écrit (aux dimensions) $: \eta V / \varepsilon^{2} \sim \rho g$, où $V$ est la vitesse moyenne de remontée de l'air. Cette vitesse est d'autant plus faible que le film est mince, à cause du quasi-blocage de l'écoulement à la frontière entre l'air qui s'écoule et l'eau environnante, 100 fois plus visqueuse : plus le film est mince, plus ce blocage concerne l'ensemble du fluide intercalé, et plus l'écoulement est lent. Cette cinétique (dite de Reynolds) a également été observée par Couder, qui a étudié l'évolution du film d'air intercalé entre une grosse goutte d'huile et le bain (identique) sur lequel elle repose [4]. Si l'antibulle claque quand elle atteint une épaisseur limite $\varepsilon_{0}$, c'est l'approche de cette épaisseur qui domine la cinétique d'amincissement. Les écoulements ayant lieu sur l'échelle $R$ de la goutte, on en déduit un temps caractéristique de drainage $\tau \sim R / V\left(\varepsilon_{0}\right) \sim \eta R / \rho g \varepsilon_{0}{ }^{2}$.

La question est donc de déterminer $\varepsilon_{0}$. Pour les bulles classiques et quand les tensioactifs sont chargés, l'épaisseur finale du film résulte de l'équilibre entre forces de van der Waals (qui tendent à amincir le film) et répulsion d'origine électrostatique : les contre-ions libérés par les têtes des tensioactifs occupent une certaine couche qu'il est défavorable (entropiquement) de confiner. On appelle films noirs ces films (méta)stables d'une dizaine de nanomètres d'épaisseur. Pour les antibulles, comme le laisse deviner le schéma $2 b$, il n'en est rien : les forces de van der Waals écrasent le film d'air sans qu'aucune barrière ne bloque le drainage ; l'épaisseur $\varepsilon_{0}$ sera donc définie par la portée des forces de van der Waals, c'est-à-dire la centaine de nanomètres. On trouve ainsi pour le temps $\tau$ une heure, au plus.
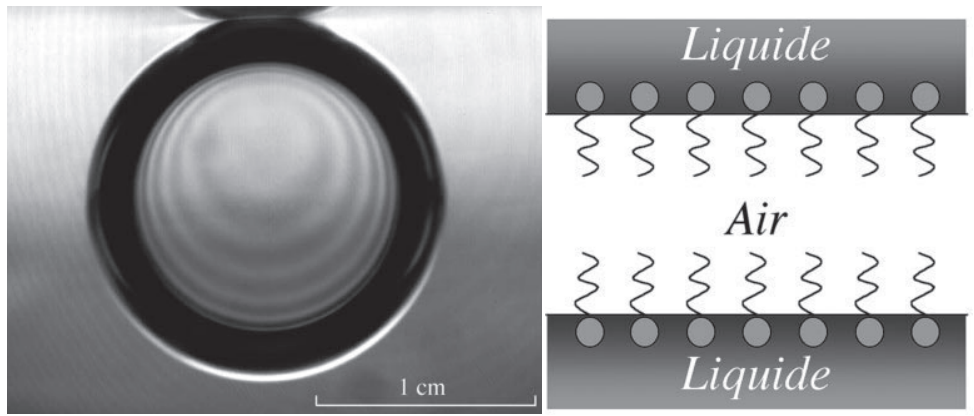

Fig. 2. (a) Photo d'une antibulle près de la surface du bain. (b) Coupe transversale du film d'air d'une antibulle de savon : la surface est couverte de molécules tensioactives dont les queues hydrophobes s'opposent.

Les durées de vie observées sont en général un peu plus courtes (inférieures à 10 minutes). II semble que ce vieillissement accéléré soit lié à l'apparition de canaux distribués selon les méridiens de la bulle : au lieu que l'écoulement soit homogène sur l'ensemble du globule, l'air est transporté du film vers les canaux (sur une courte distance, i.e. entre deux canaux) ; puis il remonte rapidement par ces conduits (plus épais), comme l'eau dégouline dans une gouttière ou dans les bordures de Plateau d'une mousse, entre deux facettes savonneuses adjacentes.

\section{Mort de l'antibulle}

L'explosion d'une antibulle peut être observée en utilisant une caméra rapide. La figure 3 montre un tel événement. Comme on l'a mentionné plus haut, l'explosion commence souvent au pôle Sud (là où le film est le plus mince). Mais le fait le plus remarquable est que le film se désagrège en plusieurs endroits à la fois.

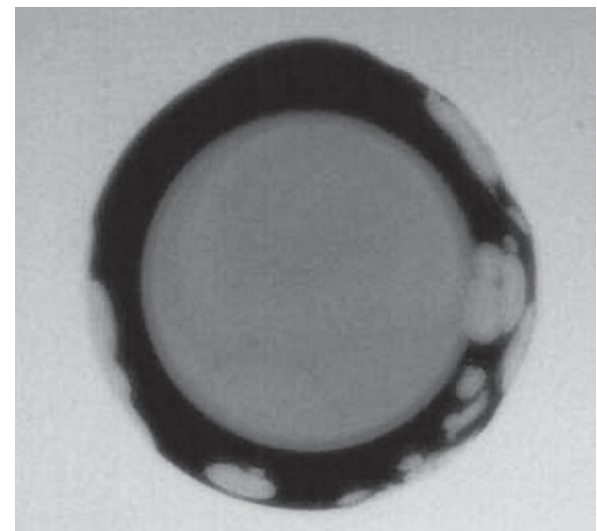

Fig. 3. Désagrégation du film d'air entourant une antibulle : le film éclate en plusieurs endroits à la fois.

une a istribution spatiale integrant laction

une distribution spatiale intégrant l'action de van der Waals (déstabilisante) et celle de la tension de surface (qui s'oppose aux fluctuations de la surface).

Le mode de rupture observé sur la figure 3 confirme donc que c'est à l'épaisseur de van der Waals que le film s'est aminci : contrairement à une bulle, rien ne stabilise alors l'antibulle. On remarquera, sur la même photographie, le renflement au pôle Nord, conséquence du drainage de l'air, qui s'est accumulé à cet endroit.

\section{Conclusion}

II semble donc que les antibulles sont des objets intrinsèquement instables, sans métastabilité possible, au contraire des bulles de savon. Au contraire de ces dernières, les contre-ions se trouvent à l'extérieur de la zone confinée, ce qui doit réduire le rôle des interactions d'origine électrostatique. Le temps de vie des antibulles résulte de la lente remontée de l'air dans le film mince qui les constitue. L'amincissement se produit jusqu'à atteindre une épaisseur de l'ordre de $100 \mathrm{~nm}$. Alors, les forces de van der Waals déstabilisent le film d'air qui se perce en de nombreux points, puis éclate. La lenteur du drainage (plusieurs minutes) fait de l'antibulle un objet intéressant pour l'encapsulation d'espèces liquides, permettant de les convoyer aisément sans mélange ni coalescence, sur des dispositifs de type microfluidique. La récupération du contenu à l'issue de leur voyage peut être activée par une impulsion de pression, ou par tout autre processus favorisant la rupture du film. La dynamique de cette rupture mériterait ellemême une étude : contrairement à ce qui se passe pour une bulle (avec qui l'antibulle s'affirme à nouveau en opposition), la cinétique de l'explosion devrait être dictée non par les propriétés du film (inertie, viscosité), mais par celles du milieu liquide extérieur (le film d'air ayant une masse et une viscosité négligeables). Notons enfin que le film lui-même peutêtre constitué d'huile, ce qui le rend beaucoup plus visqueux. Comme, en outre, le contraste de densité entre les deux fluides est alors atténué, cette variété d'antibulle saura nettement mieux résister aux effets de l'âge.

\section{Bibliographie}

[1] E. Littré, Dictionnaire de la langue française, Hachette (1873).

[2] W. Hughes et A.R. Hughes, Nature 129, 59 (1932) ; C.L. Stong, Scientific American 230, 116 (1974).

[3] Y. Amarouchène, G. Cristobal et H. Kellay, Physical Review Letters 87, 206104 (2001).

[4] Y. Couder, E. Fort, C.H. Gautier et A. Boudaoud, Physical Review Letters 94, 177801 (2005).

[5] S. Dorbolo, E. Reyssat, N. Vandewalle et D. Quéré, Europhysics Letters 69, 966, (2005).

[6] G. Reiter, Science 282, 888 (1998).

(*) S.D. remercie SNRS pour son soutien financier. 